\title{
Proposta de Instrumento para Autoavaliação de Projetos de Pesquisa Envolvendo Seres Humanos
}

\section{Proposal of an Instrument for Self-Assessment of Research Projects Involving Human Beings}

\author{
Ernandes Gonçalves Dias* \\ Departamento de Enfermagem da Faculdade Verde Norte \\ *Autor para correspondência: ernandesgdias@yahoo.com.br
}

\begin{abstract}
RESUMO
O presente relato resulta da proposta de um instrumento para autoavaliação de projetos de pesquisa que envolvem seres humanos. Foi elaborado, essencialmente, a partir da reflexão e experiência do autor em orientações de projetos de pesquisa na área de conhecimento de ciências da saúde e usado como piloto por um grupo de orientandos de um curso de graduação em Enfermagem de uma faculdade privada do Norte de Minas Gerais, no primeiro semestre de 2019. Objetiva ser uma ferramenta para diagnóstico de fragilidades em projetos de pesquisa previamente à avaliação por uma banca ou Comitê de Ética em Pesquisa, a fim de sanar eventuais problemas do projeto e favorecer a obtenção de pareceres favoráveis à realização da pesquisa. Sugere-se que o instrumento seja adotado por pesquisadores iniciantes, orientandos e orientadores de projetos de pesquisa da área de ciências sociais, humanas e da saúde, para orientar a escrita de projetos de pesquisas que envolvem seres humanos.
\end{abstract}

Palavras-Chave: Projetos de Pesquisa; Comitês de Ética em Pesquisa; Experimentação Humana.

\begin{abstract}
This report results from the proposal of an instrument for self-evaluation of research projects involving human beings. It was elaborated essentially from the author's reflection and experience in research project supervisions in the knowledge area of health science and was used as a pilot by a group of students from a nursing undergraduate course of a private college of the North of Minas Gerais in the first half of 2019. It aims to be a tool for diagnosing weaknesses in research projects before the evaluation by a research panel or Research Ethics Committee, in order to remedy any project problems and favor the obtaining of favorable opinions for the research. It is suggested that the instrument can be adopted by beginning researchers, students, and advisors of social, human and health sciences research projects to guide the writing of research projects involving human beings.
\end{abstract}

Keywords: Research Design; Ethics Committees Research; Human Experimentation.

\section{Relato do desenvolvimento do instrumento}

Quando se aproxima a conclusão de um curso de graduação, conforme regulamentado por resoluções do Ministério da Educação e Cultura (MEC), estudantes da graduação de diversos cursos, especialmente da área de ciências sociais, humanas e da saúde, se deparam com a necessidade de escrever o projeto de Trabalho de Conclusão de Curso (TCG).

Nesse momento é comum observar os estudantes vivenciarem sentimentos de medo, incerteza, insegurança, ansiedade e dúvidas em relação à construção do projeto de pesquisa.

Outras dificuldades colocadas pelos estudantes sobre a construção do projeto de pesquisa estão relacionadas com a escrita do referencial teórico, bibliografia e dificuldades relacionadas com o método científico (FREITAS et al., 2013).

Sabe-se que durante a graduação, principalmente, acadêmicos de faculdades e universidades particulares do Brasil têm pouco ou nenhum envolvimento com pesquisa, sendo, em muitos 
casos, o primeiro contato com a elaboração de um projeto de pesquisa, o que ocorre já no momento da escrita do TCG. Isso representa um gargalo do Ensino Superior privado no Brasil, o qual Almeida et al. (2018) confirmam pela afirmação de que as atividades de pesquisa realizadas no ensino superior em instituição privada ainda são discretas.

Corrobora esses argumentos o fato de que as universidades públicas estaduais e federais respondem por mais de 95\% da produção científica do Brasil (MOURA, 2019), então é plausível a construção deste instrumento (Quadro 1) para estudantes de faculdades e universidades particulares, e até mesmo para os estudantes iniciantes em pesquisa de instituições de ensino públicas, visto que tem como objetivo ser um meio de os estudantes de graduação autoavaliarem seu projeto de pesquisa, antes de darem sequência às próximas etapas da pesquisa.

$\mathrm{O}$ instrumento ora proposto foi construído a partir da reflexão e experiência do autor em orientações de projetos de pesquisa em nível de graduação e pós-graduação lato sensu na área de conhecimento de ciências da saúde, da consulta a blogs, da opinião de especialistas e de outros materiais disponíveis na literatura.

Os projetos de pesquisa que envolvem direta ou indiretamente seres humanos, inclusive na modalidade de TCG, devem, obrigatoriamente, ser avaliados por um Comitê de Ética em Pesquisa (CEP) (BRASIL, 2012; BRASIL, 2016).

Assim sendo, a relação entre a ética e a pesquisa envolvendo seres humanos presente no instrumento se dá ao passo que este, apesar de permitir autoavaliação global do projeto de pesquisa, contempla elementos que são essenciais em projetos com proposta de intervenções diretas ou indiretas em seres humanos e que, por isso, necessitam de apreciação ética por um CEP.

Dessa forma, a intenção é que o instrumento subsidie os pesquisadores iniciantes, que estão diante da necessidade de produzir um projeto de pesquisa com qualidade, consistência e que apresente o rigor e os elementos necessários para posterior julgamento em banca, conforme regulamento do curso e submissão e avaliação por um CEP.
$\mathrm{Na}$ elaboração do instrumento consideraram-se como norteadoras as normas da Associação Brasileira de Normas Técnicas (ABNT) e a cronologia de organização de um projeto de pesquisa. Está estruturado em quatro categorias: elementos pré-textuais, elementos textuais, elementos pós-textuais e aspectos gerais, com um campo para observações. As categorias elementos textuais e pós-textuais são constituídas também por subcategorias para melhor organizar o conteúdo.

Nas categorias e subcategorias há questionamentos em relação à estrutura, escrita, formatação e conteúdo, elementos importantes que estejam presentes em um projeto de pesquisa. Laterais aos questionamentos estão, em forma de check-list, as possibilidades de resposta para avaliação de um item em questão; as possibilidades apresentadas são: sim, não ou não se aplica (N/A).

Para cada questionamento admite-se somente uma opção de resposta. Comentários sobre itens em análise podem ser registrados no campo "Observações", presente no final do instrumento.

A escrita adotada na construção do instrumento é bastante simples para que qualquer estudante de graduação tenha condições de aplicá-lo e consiga realizar uma autoavaliação crítica e diagnóstico eficaz em seu projeto de pesquisa. Assim, recomenda-se o uso do instrumento minimamente em três momentos: durante a fase de elaboração do projeto; após a conclusão da escrita (antes da pré-banca, se existir) e antes da submissão do projeto na Plataforma Brasil, a fim de que as fragilidades identificadas sejam sanadas.

O instrumento foi usado como piloto por graduandos em Enfermagem de uma Instituição de Ensino Superior particular do Norte de Minas Gerais no primeiro semestre de 2019 e se mostrou eficiente para os orientandos autoavaliarem e identificarem fragilidades presentes no projeto de pesquisa. Essa experiência possibilitou adequações para delinear e tornar o projeto mais robusto, especialmente no tocante ao método, em que apresenta informações fundamentais para o CEP julgar o cumprimento das diretrizes éticas que envolvem a pesquisa com seres humanos. 
Apesar das argumentações, esta proposta não pretende "engessar" a criatividade dos estudantes de graduação na elaboração de seus projetos de pesquisa, nem esgotar o tema. Este instrumento é uma opção complementar às orientações recebidas de um orientador, a consultas às normas da ABNT e ao manual de normas da instituição, se houver.

No entanto, a disponibilização deste instrumento de autoavaliação para aplicação em projetos de pesquisa é uma produção inovadora, visto que não há registros de outros instrumentos de tal natureza publicados no Brasil.

O uso do instrumento poderá partir do próprio orientando, na intenção de melhorar seu projeto de pesquisa, ou adotado por um orientador como ferramenta de avaliação parcial do desenvolvimento acadêmico e do projeto de pesquisa de um orientando. Espera-se que com o uso deste instrumento seja possível identificar fragilidades do projeto de pesquisa previamente à avaliação por uma banca ou por um CEP, o que evitará que o projeto retorne para correções e favorecerá a obtenção de pareceres favoráveis ao desenvolvimento da pesquisa.

Ademais, acredita-se que projetos de pesquisa bem escritos e estruturados certamente mostram desenvolvimento, capacidade intelectual e amadurecimento do pesquisador, além de, hipoteticamente, indicarem que a pesquisa será mais bem conduzida e terá maior potencial para produzir novos conhecimentos.

O quadro 1, apresentado na sequência, expõe o instrumento proposto para subsidiar, especialmente a pesquisadores iniciantes, a autoavaliação em projetos de pesquisa que envolvam seres humanos, conforme argumentado.

\begin{tabular}{|c|c|c|c|c|}
\hline \multirow{2}{*}{ ITEM } & \multirow{2}{*}{ DESCRIÇÃO } & \multicolumn{3}{|c|}{ Avaliação } \\
\hline & & SIM & NÃO & $\mathrm{N} / \mathrm{A}$ \\
\hline \multirow{9}{*}{$\begin{array}{l}\text { ELEMENTOS } \\
\text { PRÉ-TEXTUAIS }\end{array}$} & Há capa conforme norma? & & & \\
\hline & $\begin{array}{l}\text { O título do trabalho é claro, objetivo e } \\
\text { tem relação com o objetivo e problema da } \\
\text { pesquisa? }\end{array}$ & & & \\
\hline & Há folha de rosto conforme norma? & & & \\
\hline & Há folha de avaliação conforme norma? & & & \\
\hline & $\begin{array}{l}\text { Os nomes dos autores aparecem comple- } \\
\text { tos, em ordem alfabética na capa, folha de } \\
\text { rosto e folha de aprovação, se houver? }\end{array}$ & & & \\
\hline & $\begin{array}{l}\text { Há lista de siglas/abreviaturas correspon- } \\
\text { dentes ao conteúdo do trabalho? }\end{array}$ & & & \\
\hline & $\begin{array}{l}\text { Há resumo da proposta (caráter informa- } \\
\text { tivo, parágrafo único, sem enumeração de } \\
\text { tópicos abordando tema geral, justifica- } \\
\text { tiva e problema da pesquisa; objetivos e } \\
\text { métodos)? }\end{array}$ & & & \\
\hline & Há descritores/palavras-chave? & & & \\
\hline & $\begin{array}{l}\text { O sumário corresponde à paginação e ao } \\
\text { conteúdo do trabalho? }\end{array}$ & & & \\
\hline
\end{tabular}




\begin{tabular}{|c|c|c|c|c|c|c|}
\hline & \multirow{2}{*}{\multicolumn{2}{|c|}{ ITEM }} & \multirow{2}{*}{ DESCRIÇÃO } & \multicolumn{3}{|c|}{ Avaliação } \\
\hline & & & & \multirow[t]{2}{*}{ SIM } & \multirow[t]{2}{*}{ NÃO } & \multirow[t]{2}{*}{$\mathrm{N} / \mathrm{A}$} \\
\hline \multirow{26}{*}{ 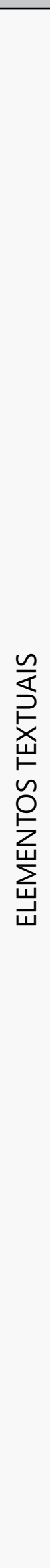 } & \multirow{2}{*}{\multicolumn{2}{|c|}{ INTRODUÇÃO }} & A introdução é clara, contextualizada e atual? & & & \\
\hline & & & Apresenta a questão problema? & & & \\
\hline & \multirow{6}{*}{\multicolumn{2}{|c|}{ JUSTIFICATIVA }} & $\begin{array}{l}\text { A justificativa é clara, contextualiza o pro- } \\
\text { blema com as evidências científicas? }\end{array}$ & & & \\
\hline & & & $\begin{array}{l}\text { A justificativa é convincente e demonstra } \\
\text { relevância para pesquisa? }\end{array}$ & & & \\
\hline & & & $\begin{array}{l}\text { A justificativa demonstra a relevância cien- } \\
\text { tífica da pesquisa? }\end{array}$ & & & \\
\hline & & & $\begin{array}{l}\text { A justificativa demonstra a relevância so- } \\
\text { cial da pesquisa? }\end{array}$ & & & \\
\hline & & & $\begin{array}{l}\text { Aponta perspectivas nas áreas acadêmicas, } \\
\text { tecnológica, científica, filosófica ou social? }\end{array}$ & & & \\
\hline & & & $\begin{array}{l}\text { É clara a motivação dos pesquisadores } \\
\text { pela abordagem realizada? }\end{array}$ & & & \\
\hline & \multirow{6}{*}{\multicolumn{2}{|c|}{ OBJETIVOS }} & O objetivo geral é claro e consistente? & & & \\
\hline & & & $\begin{array}{l}\text { O objetivo geral condiz com a questão } \\
\text { problema de pesquisa? }\end{array}$ & & & \\
\hline & & & $\begin{array}{l}\text { O objetivo geral é coerente com o título } \\
\text { do projeto? }\end{array}$ & & & \\
\hline & & & $\begin{array}{l}\text { Os objetivos específicos são claros e bem } \\
\text { definidos? }\end{array}$ & & & \\
\hline & & & $\begin{array}{l}\text { Os objetivos específicos contribuem para } \\
\text { o alcance do objetivo geral? }\end{array}$ & & & \\
\hline & & & $\begin{array}{l}\text { Os objetivos são afirmados com verbos no } \\
\text { infinitivo? }\end{array}$ & & & \\
\hline & \multirow{6}{*}{\multicolumn{2}{|c|}{ FUNDAMENTAÇÃO* }} & $\begin{array}{l}\text { Dá suporte/sustentação para elucidar o } \\
\text { problema de pesquisa? }\end{array}$ & & & \\
\hline & & & $\begin{array}{l}\text { É contextualizada com o problema e com } \\
\text { os objetivos da pesquisa? }\end{array}$ & & & \\
\hline & & & É atual e consistente? & & & \\
\hline & & & Define conceitos essenciais para a pesquisa? & & & \\
\hline & & & $\begin{array}{l}\text { Os tópicos são organizados em sequência } \\
\text { lógica/coerente? }\end{array}$ & & & \\
\hline & & & Utiliza autores de renome da temática? & & & \\
\hline & \multirow{6}{*}{ 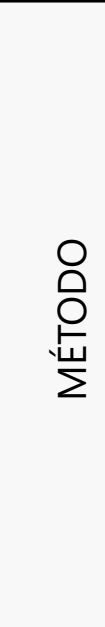 } & \multirow[b]{2}{*}{ TIPO } & Delimita o tipo de pesquisa? & & & \\
\hline & & & $\begin{array}{l}\text { O tipo de pesquisa é conceitualmente } \\
\text { justificado? }\end{array}$ & & & \\
\hline & & \multirow{2}{*}{$\begin{array}{l}\text { POPU- } \\
\text { LAÇÃO }\end{array}$} & $\begin{array}{l}\text { A população/universo da pesquisa é afir- } \\
\text { mada de forma clara e pontual? }\end{array}$ & & & \\
\hline & & & $\begin{array}{l}\text { Apresenta e desenvolve as características } \\
\text { da população/universo de pesquisa? }\end{array}$ & & & \\
\hline & & \multirow{2}{*}{$\begin{array}{l}\text { AMOSTRA } \\
\text { INFOR- } \\
\text { MANTES }\end{array}$} & $\begin{array}{l}\text { Há amostra/informantes para pesquisa? } \\
\text { Se amostra, o cálculo é fundamentado? É } \\
\text { representativa? }\end{array}$ & & & \\
\hline & & & $\begin{array}{l}\text { Há critérios de inclusão/exclusão ou sele- } \\
\text { ção claros, éticos e aplicáveis? }\end{array}$ & & & \\
\hline
\end{tabular}




\begin{tabular}{|c|c|c|c|c|c|c|}
\hline \multirow{2}{*}{\multicolumn{3}{|c|}{ ITEM }} & \multirow{2}{*}{ DESCRIÇÃO } & \multicolumn{3}{|c|}{ Avaliação } \\
\hline & & & & \multirow[t]{2}{*}{ SIM } & \multirow[t]{2}{*}{ NÃO } & \multirow[t]{2}{*}{ N/A } \\
\hline \multirow{27}{*}{ 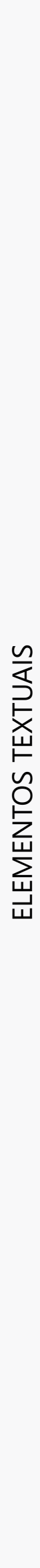 } & \multirow{13}{*}{\multicolumn{2}{|c|}{ 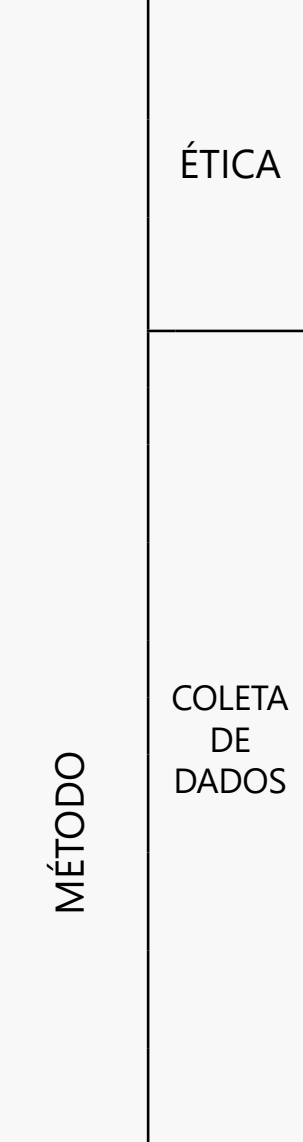 }} & $\begin{array}{l}\text { Há previsão de cumprimento de aspectos éti- } \\
\text { cos (anonimato, sigilo, confidencialidade etc.)? }\end{array}$ & & & \\
\hline & & & $\begin{array}{l}\text { Cita Resolução } 466 / 2012 \text { ou } 510 / 2016 \text { ou ou- } \\
\text { tra legislação vigente sobre ética em pesquisa? }\end{array}$ & & & \\
\hline & & & $\begin{array}{l}\text { Há indicação de submissão do projeto } \\
\text { para avaliação ética por um CEP? }\end{array}$ & & & \\
\hline & & & Há técnica para coleta de dados? & & & \\
\hline & & & Há instrumento para coleta de dados? & & & \\
\hline & & & $\begin{array}{l}\text { Há descrição da elaboração/adaptação do } \\
\text { instrumento de coleta de dados? }\end{array}$ & & & \\
\hline & & & $\begin{array}{l}\text { Há previsão de quando os dados serão } \\
\text { coletados? }\end{array}$ & & & \\
\hline & & & Há previsão de quem coletará os dados? & & & \\
\hline & & & Há previsão de local para coleta de dados? & & & \\
\hline & & & $\begin{array}{l}\text { Há previsão de quantidade de tentativas } \\
\text { de coleta de dados? }\end{array}$ & & & \\
\hline & & & $\begin{array}{l}\text { Há previsão de momento/tempo/período } \\
\text { para encerramento da coleta de dados? }\end{array}$ & & & \\
\hline & & & $\begin{array}{l}\text { Há previsão de duração, em tempo, para } \\
\text { coleta de dados? }\end{array}$ & & & \\
\hline & & & $\begin{array}{l}\text { Há previsão de meio/instrumento para } \\
\text { registro dos dados coletados? }\end{array}$ & & & \\
\hline & & \multirow{3}{*}{$\begin{array}{l}\text { ANÁLISE/ } \\
\text { DADOS }\end{array}$} & $\begin{array}{l}\text { Há previsão de como será a organização } \\
\text { dos dados coletados? }\end{array}$ & & & \\
\hline & & & $\begin{array}{l}\text { Há previsão de método estatístico/teórico } \\
\text { para guiar análise dos dados? }\end{array}$ & & & \\
\hline & & & $\begin{array}{l}\text { Há previsão da forma de apresentação dos } \\
\text { dados? Garante anonimato dos informantes? }\end{array}$ & & & \\
\hline & & \multirow{3}{*}{$\begin{array}{c}\text { AV. } \\
\text { GERAL } \\
\text { DO } \\
\text { MÉTODO }\end{array}$} & O método é claro e objetivo? & & & \\
\hline & & & O método permite responder aos objetivos? & & & \\
\hline & & & Apresenta possibilidade de reprodução? & & & \\
\hline & \multirow{4}{*}{\multicolumn{2}{|c|}{ CRONOGRAMA }} & Há cronograma? & & & \\
\hline & & & Prevê etapas e prazo de cumprimento? & & & \\
\hline & & & $\begin{array}{l}\text { As etapas previstas são coerentes com o } \\
\text { projeto? }\end{array}$ & & & \\
\hline & & & $\begin{array}{l}\text { O tempo de cumprimento das etapas é } \\
\text { pertinente? }\end{array}$ & & & \\
\hline & \multirow{4}{*}{\multicolumn{2}{|c|}{ ORÇAMENTO }} & Há orçamento? & & & \\
\hline & & & $\begin{array}{l}\text { O orçamento apresentado é coerente com } \\
\text { os gastos prováveis? }\end{array}$ & & & \\
\hline & & & $\begin{array}{l}\text { Há demonstração de responsáveis pelo } \\
\text { custeio da pesquisa? }\end{array}$ & & & \\
\hline & & & $\begin{array}{l}\text { A natureza dos gastos é discriminada no } \\
\text { orçamento? }\end{array}$ & & & \\
\hline
\end{tabular}




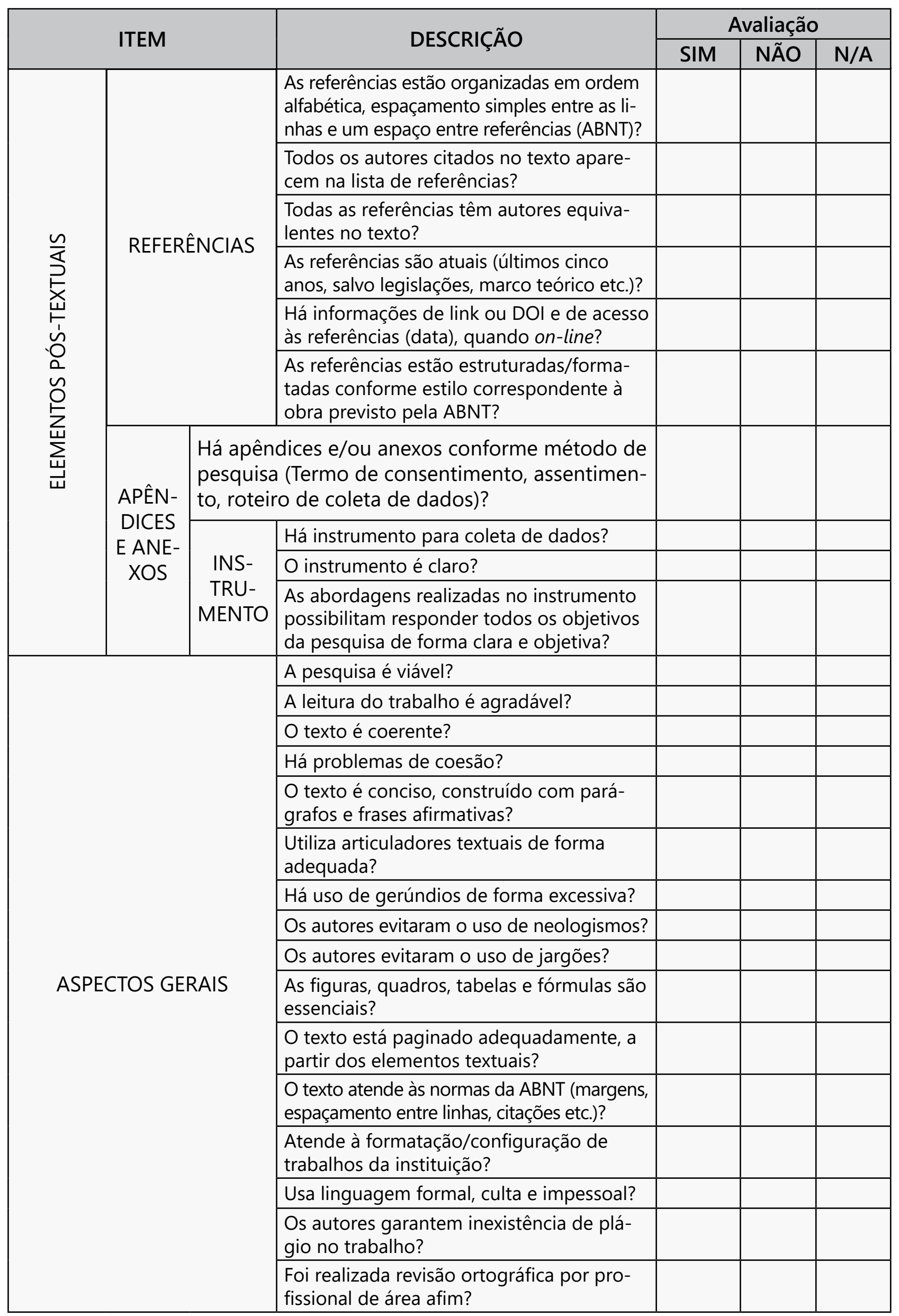




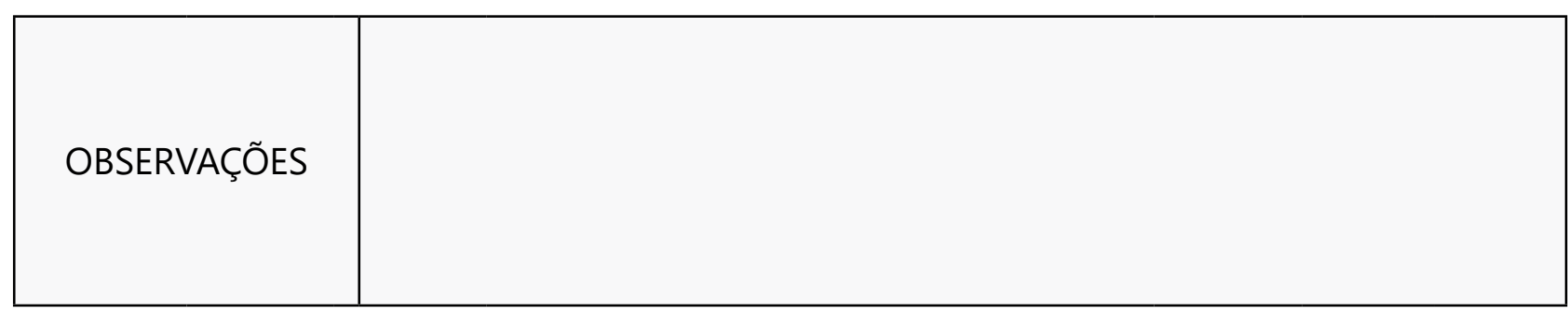

de de

\section{Assinatura do Orientando}

*É possível que os elementos deste tópico sejam estruturados dentro da Introdução e o tópico Fundamentação não exista em um projeto de pesquisa.

Quadro 1 - Check-List de autoavaliação em projetos de pesquisa envolvendo seres humanos.

\section{Referências Bibliográficas}

ALMEIDA, Genésio Lopes Mercês de et al. "Iniciação Científica por Meio da Pesquisa Experimental In Vivo: Relato de Experiência Acadêmica". Rev. Grad. USP, vol. 3, n. 2, jul. 2018, pp. 93-97. Disponível em: $<$ https://doi.org/10.11606/issn.2525-376X.v3i2p93-97>. Acessado em 18 ago. 2019.

BRASIL. Ministério da Saúde. Conselho Nacional de Saúde. "Resolução 466 de 12 de dezembro de 2012". Brasília, DF. 2012. Disponível em: \http://bvsms.saude. gov.br/bvs/saudelegis/cns/2013/res0466_12_12_2012. html $>$. Acessado em 5 jul. 2019.

BRASIL. Ministério da Saúde. Conselho Nacional de Saúde. "Resolução 510 de 7 de abril de 2016. Brasília", DF. 2016. Disponível em: 〈http://bvsms.saude.gov.br/ bvs/saudelegis/cns/2016/res0510_07_04_2016.html >. Acessado em 5 jul. 2019.
FREITAS, Samille Maria Bandeira et al. "Dificuldades Vivenciadas na Construção do TCC: Percepção de Estudantes Egressos de um Curso de Graduação em Enfermagem". $17^{\circ}$ Seminário Nacional de Pesquisa em Enfermagem (SENPE). O Clássico e o emergente: desafios da pesquisa em Enfermagem. Hotel Praia Mar, Natal, RN, 3 a 5 de junho de 2013. Disponível em: $<$ http://www.abeneventos.com.br/anais_senpe/17senpe/ pdf/1782co.pd† $\gg$. Acessado em 7 jul. 2019.

MOURA, Mariluce. "Universidades Públicas Respondem por Mais de 95\% da Produção Científica do Brasil". Blog, Politica Cientifica e Tecnológica, Reportagens e Artigos. Ciência na Rua, 2019. Disponível em: >http:// ciencianarua.net/universidades-publicas-respondem-por-mais-de-95-da-producao-cientifica-do-brasil/ $>$. Acessado em 9 jul. 2019.

Publicado em 28/07/2020. 